\title{
EXTRACTING DEM FROM AIRBORNE X-BAND DATA BASED ON POLINSAR
}

\author{
Xiuxiu Hou ${ }^{\mathrm{a}, *}$, Guoman Huang ${ }^{\mathrm{a}}$, Zheng Zhao, \\ ${ }^{\text {a }}$ Chinese Academy of surveying and Mapping, Beijing, China - sdkd_hxx@126.com
}

Commission VI, WG VI/4

KEY WORDS: Polarimetric Interferometric Synthetic Aperture Radar, DEM, Coherence optimization, Airborne X-band data

\begin{abstract}
:
Polarimetric Interferometric Synthetic Aperture Radar (PolInSAR) is a new trend of SAR remote sensing technology which combined polarized multichannel information and Interferometric information. It is of great significance for extracting DEM in some regions with low precision of DEM such as vegetation coverage area and building concentrated area. In this paper we describe our experiments with high-resolution X-band full Polarimetric SAR data acquired by a dual-baseline interferometric airborne SAR system over an area of Danling in southern China. Pauli algorithm is used to generate the double polarimetric interferometry data, Singular Value Decomposition (SVD), Numerical Radius (NR) and Phase diversity (PD) methods are used to generate the full polarimetric interferometry data. Then we can make use of the polarimetric interferometric information to extract DEM with processing of pre filtering, image registration, image resampling, coherence optimization, multilook processing, flat-earth removal, interferogram filtering, phase unwrapping, parameter calibration, height derivation and geo-coding. The processing system named SARPlore has been exploited based on VC++ led by Chinese Academy of Surveying and Mapping. Finally compared optimization results with the single polarimetric interferometry, it has been observed that optimization ways can reduce the interferometric noise and the phase unwrapping residuals, and improve the precision of DEM. The result of full polarimetric interferometry is better than double polarimetric interferometry. Meanwhile, in different terrain, the result of full polarimetric interferometry will have a different degree of increase.
\end{abstract}

\section{INTRODUCTION}

As a kind of active sensor, Synthetic Aperture Radar (SAR) not only can realize all day, all-weather observation without restriction of lighting and weather conditions, but also can get the information of the earth's surface and subsurface of vegetation, these characteristics make it in agriculture, forestry, geology, environment, hydrology, Marine, disasters, the application of surveying and mapping and military field has a unique advantage. After a long period of development, Synthetic Aperture Radar technology from a single band, single polarization has gradually developed to the multiband, full polarization. The presence of Polarimetric Interferometric Synthetic Aperture Radar (PolInSAR) has makes the SAR remote sensing application to a climax. PolInSAR realized the observation space's expansion by the effective combination of interference information and polarization information, that makes it has both InSAR's sensitivity to the spatial distribution of vegetation scattering body features, but also has PolSAR's sensitivity to the shape and direction of vegetation scattering body features. Therefore, PolInSAR technology can separate the feature components from different height and different scattering mechanism component to determine the coherence with the highest weight for phase difference estimation.

An important application field of PolInSAR is high precision Digital Elevation Model (DEM) generation. Polarization coherent interference optimal process can effectively reduce the decorrelation caused by scattering center height difference in an effective resolution cell, especially in the area of the surface vegetation, the correlation is more serious. So the polarization interference coherent optimal process can effectively eliminate the residual decorrelation caused by scattering component, thus greatly improve the coherence. Therefore, DEM precision is generated by the PolInSAR can be significantly improved, at present a lot of research also proves this point: Papathanassiou (Cloude, 1997) makes use of the SIR - C/X SAR data to study the influence to coherence of frequency and polarization, this is the origin of introducing polarization to InSAR. After that polarization interference coherent optimal algorithm and the target decomposition theory based on the coherent optimal are proposed, which laid the theoretical basis of the PolInSAR; Li Xinw (Li Xinwu, 2002) and (Li Xinwu,2002) etc. based on the polarization interference SIR - C L-band SAR data, using the Singular Value Decomposition (SVD) to extract DEM, found that the optimization method is better than the traditional VV polarization channels, especially in the vegetation coverage, the improvement of DEM accuracy is about $10 \mathrm{~m}$; Xiong Tao (Xiong Tao,2007), polarization interference information and information fusion method is utilized to extract DEM, found a $7 \%$ increase over the phase after the fusion quality, phase unwrapping bound residues almost have reduced more than 95\%; In addition, Brandafss (Brandfass,2002) uses $\mathrm{P}$ wave polarization interference SAR study on the DEM extraction in forest areas, Lucina(Luciano V D,2002)and others use $\mathrm{P}$ and $\mathrm{x}$ bands in forest areas to make a similar study on DEM extraction, all of them make it that DEM extraction accuracy has improved significantly.

The sources of the polarization interference measurement data are mainly short baseline shuttle SAR repeat pass interference and interference of repeat pass airborne SAR and long return cycle spaceborne polarization interference SAR. Double antennas airborne SAR data reduce the influence of time to coherent, make coherence interference greatly enhanced. In this

\footnotetext{
${ }^{*}$ Corresponding author
} 
case, is the polarization interference still superior to the conventional interferometry method? Are different optimization methods and different polarization channels on the improvement of the DEM precision consistent? In this paper we describe our experiments with high-resolution X-band full Polarimetric SAR data acquired by a dual-baseline interferometric airborne SAR system over an area of Danling in southern China. Pauli algorithm is used to generate the double polarimetric interferometry data, Singular Value Decomposition (SVD), Numerical Radius (NR) and Phase diversity (PD) methods are used to generate the full polarimetric interferometry data. And the results of the accuracy of DEM extraction are analyzed.

\section{POLARIZATION INTERFERENCE MEASURING PRINCIPLE}

\subsection{The scattering matrix of the vector interference}

The conventional InSAR technology uses the two SAR complex images from the different perspective view to do coherent processing with complex conjugate multiplication. Then interferometric phase is transformed into the elevation information. Based on dual-baseline interferometric airborne SAR as the research object, the following to make cross - track interferometry (XTI) model as an example to introduce the InSAR height measurement. XTI is equipped with two vertical flight direction of antenna on flight platform, if baseline fixed and can obtain high precision platform's position, we can obtain accurate surface $3 \mathrm{~d}$ information.

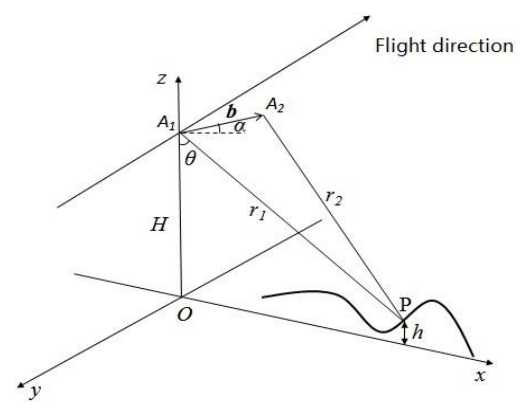

Figure 1. Dual-baseline airborne InSAR height measurement geometry relationship

As shown in figure $1, \mathrm{~A}_{1}$ and $\mathrm{A}_{2}$ are two antennas for airborne platform, including standard operating mode and ping-pong model. Standard model is single-emission and double-receiving, or main antenna $A_{1}$ is responsible for transmitting and receiving radar signal, the auxiliary antenna $\mathrm{A}_{2}$ will receive only the radar echo signal; Ping-pong model is double-emission and doublereceiving, that is, two antenna launch reception in turn. $B$ is the baseline vector, ais baseline orientation, $\theta$ is Angle of view, $r_{1} 、 r_{2}$ respectively to the slant distance of point $P$. So:

$h=H-r_{1}\left(\alpha-\arcsin \left(\frac{\left(\frac{\lambda \varphi}{2 \pi Q}+r_{1}\right)^{2}-r_{1}^{2}-b^{2}}{2 r_{1} \cdot b}\right)\right)$

where $\lambda=$ Radar wavelength

$$
\mathrm{Q}: \text { standard operating mode }=1 \text {, ping-pong } \text { model }=2 .
$$

Conventional SAR interference's coherence is defined as the absolute value of the plural of normalized cross-correlation:

$$
\gamma_{\text {Int }}=\frac{\left|\left\langle s_{1} s_{2}^{*}\right\rangle\right|}{\sqrt{\left\langle s_{1} s_{1}^{*}\right\rangle\left\langle s_{2} s_{2}^{*}\right\rangle}}
$$

where $\quad 0 \leq \gamma \leq 1$

PolInSAR coherence definition defined by extending the conventional SAR interference :

$$
\gamma\left(\omega_{1}, \omega_{2}\right)=\frac{\left|\left(\omega_{1}^{* T} \Omega_{12} \omega_{2}\right)\right|}{\sqrt{\left(\omega_{1}^{* T} T_{11} \omega_{1}\right)\left(\omega_{2}^{* T} T_{22} \omega_{2}\right)}}
$$

where $\omega_{1}, \omega_{2}=$ the complex projection vector;

$$
\begin{aligned}
& \mathrm{T}_{11}=\left\langle\mathrm{k}_{1} \mathrm{k}_{1}^{* \mathrm{~T}}\right\rangle, \mathrm{T}_{22}=\left\langle\mathrm{k}_{2} \mathrm{k}_{2}^{* \mathrm{~T}}\right\rangle, \Omega_{12}=\left\langle\mathrm{k}_{1} \mathrm{k}_{2}^{* \mathrm{~T}}\right\rangle ; \\
& \mathrm{k}_{1}=\mathrm{V}\left(\left[\mathrm{S}_{1}\right]\right)=\frac{1}{\sqrt{2}}\left[\mathrm{~S}_{\mathrm{HH}_{1}}+\mathrm{S}_{\mathrm{VV}_{1}}, \mathrm{~S}_{\mathrm{HH}_{1}}-\mathrm{S}_{\mathrm{VV}_{1}}, 2 \mathrm{~S}_{\mathrm{HV}_{1}}\right]^{\mathrm{T}} \\
& \mathrm{k}_{2}=\mathrm{V}\left(\left[\mathrm{S}_{2}\right]\right)= \\
& \frac{1}{\sqrt{2}}\left[\mathrm{~S}_{\mathrm{HH}_{2}}+\mathrm{S}_{\mathrm{VV}_{2}}, \mathrm{~S}_{\mathrm{HH}_{2}}-\mathrm{S}_{\mathrm{VV}_{2}}, 2 \mathrm{~S}_{\mathrm{HV}_{2}}\right]^{\mathrm{T}}
\end{aligned}
$$

\subsection{Polarization interference optimization method}

The process of extracting DEM can be divided into pre filtering, image registration, image resampling, coherence optimization, multilook processing, flat-earth removal, interferogram filtering, phase unwrapping, parameter calibration, height derivation and geo-coding. Compared with the traditional InSAR processing, the coherent optimization is additional. Coherent optimization mainly put the polarization multimodal information fusion to the interference information by the vector method and optimizing the interference to extract more accurate phase information. The current research methods mainly divided into three: Singular Value Decomposition (SVD), Numerical Radius (NR) and Phase diversity (PD).

2.2.1 SVD: Cloude (Cloude, 1997) and (Cloude,2003) descripted the method of the SVD in detail, specific idea is: make the denominator remains unchanged, maximize the molecules. Specific expression is as follows:

$$
L_{S}=\omega_{1}^{\mathrm{H}}\left[\Omega_{12}\right] \omega_{2}+\lambda_{1}\left(\omega_{1}^{\mathrm{H}}\left[\Omega_{11}\right] \omega_{1}-C_{1}\right)+\lambda_{2}\left(\omega_{2}^{\mathrm{H}}\left[\Omega_{22}\right] \omega_{2}-C_{2}\right)
$$

Make $L_{S}$ of four variables $\lambda_{1} 、 \lambda_{2} 、 \omega_{1} 、 \omega_{2}$ of partial derivative is zero; we can get the following equation to solve the eigenvalue and eigenvector:

$$
\begin{aligned}
& {\left[\mathrm{T}_{11}\right]^{-1}\left[\Omega_{12}\right]\left[\mathrm{T}_{22}\right]^{-1}\left[\Omega_{12}\right]^{\mathrm{H}} \omega_{1}=\lambda_{1} \lambda_{2}^{*} \omega_{1},} \\
& {\left[\mathrm{~T}_{22}\right]^{-1}\left[\Omega_{12}\right]^{\mathrm{H}}\left[\mathrm{T}_{11}\right]^{-1}\left[\Omega_{12}\right] \omega_{1}=\lambda_{1} \lambda_{2}^{*} \omega_{2} .}
\end{aligned}
$$

At this point, the coherence of the optimal problem was converted to SVD decomposition method to solve the eigenvalue and eigenvector of the biggest problems, through the decomposition we can get 3 set of eigenvalues and eigenvectors, Characteristic value through the type (3) can respectively get the interference information of SVD1, SVD2, and SVD3.

2.2.1 NR: The method of NR (Colin E, 2006) ask for projection vector $\omega_{1} 、 \omega_{2}$ must be the same, make $\omega_{1}=\omega_{2}=\omega$, 
and make $\mathrm{T}=\frac{\left[\mathrm{T}_{11}\right]+\left[\mathrm{T}_{22}\right]}{2}$ replace $\left[\mathrm{T}_{11}\right] 、\left[\mathrm{~T}_{12}\right]$, this method is equivalent to solve numerical radius of $\Pi=\mathrm{T}^{-1 / 2}\left[\Omega_{12}\right] \mathrm{T}^{-1 / 2}$, Method is as follows:

(1) Define the matrix $\prod$ numerical range: $\mathrm{F}(\Pi)$ $=\left\{x^{H} \Omega x\right\}, x \in C^{3}$ and $x^{H} X=1$, numerical radius: $\mathrm{r}$ (П) $=\max \{|\mathrm{F}(\Pi)|\}$.

In order to make the local optimal extension to the global optimal, import $\mathrm{e}^{\mathrm{i} \theta_{k}}$, and choose the different initial Angle $\theta_{10}, \theta_{20}, \theta_{30}$ to substitute the matrix $\mathrm{H}_{\theta_{\mathrm{K}}}=\left(\prod^{\mathrm{i} \theta^{\mathrm{k}}}+\Pi^{\dagger} \mathrm{e}^{-\mathrm{i} \theta^{\mathrm{k}}}\right) / 2, \theta_{\mathrm{k}}=\theta_{10}$ 、

$\theta_{20} 、 \theta_{30}$, calculate the largest eigenvalue lambda $\lambda_{\operatorname{maxk}}$ and the corresponding eigenvector $\chi_{\mathrm{k}}$ of $\mathrm{H}_{\Theta_{\mathrm{K}}}, \uparrow$ is the generalized inverse matrix.

Define $\theta_{\mathrm{k}}{ }^{\prime}=\arg \left\{\mathrm{x}^{\mathrm{H}} \mathrm{H}_{\theta_{\mathrm{K}}} \mathrm{x}\right\}$, if $\left|\theta_{\mathrm{k}}-\theta_{\mathrm{k}}{ }^{\prime}\right|<\varepsilon, \varepsilon$ is a small constant, then the characteristic vector $\chi_{k}$ is the best, otherwise make $\theta_{\mathrm{k}+1}=-\theta_{\mathrm{k}}{ }^{\prime}$ in step (2), (3) iterative, until find meet the conditions of three groups of feature vector.

By this method, you can see that three initial iteration points can get three characteristic vectors, by formula (3) can respectively get NR1, NR2 and NR3 three interference information.

2.2.1 PD: The method of PD (Tabb M, 2002) by maximizing the cotangent function for optimization of the eigenvalues and eigenvectors, Cotangent function form is as follows:

$$
\cot (\tilde{\gamma})=\frac{\operatorname{Re}\{\tilde{\gamma}\}}{\operatorname{Im}\{\tilde{\gamma}\}}=\frac{\omega^{\dagger}\left(\left[\Omega_{12}\right]+\left[\Omega_{12}\right]^{\dagger}\right) \omega}{\omega^{\dagger}\left(-i\left[\Omega_{12}\right]-\left[\Omega_{12}\right]^{\dagger}\right) \omega}
$$

Specific algorithm is as follows:

$$
\begin{gathered}
\text { The }\left[\Omega_{12}\right] \text { do a phase rotation transform, we can } \\
\text { get }\left[\widetilde{\Omega}_{12}\right]=\left[\Omega_{12}\right] e^{i \varphi}, \varphi=\frac{\pi}{2}-\angle \operatorname{tr}\left(\left[\Omega_{12}\right]\right) \text { 。 } \\
\text { Calculate matrix: }\left(\left[\Omega_{12}\right]+\left[\Omega_{12}\right]^{\dagger}\right) \text {, - } \\
\text { (2) }\left(\left[\Omega_{12}\right]-\left[\Omega_{12}\right]^{\dagger}\right) \text {. } \\
\text { To solve the eigenvalue and eigenvector of } \\
\text { (3) }\left(\left[\Omega_{12}\right]+\left[\Omega_{12}\right]^{\dagger}\right) \omega=-\mathrm{i} \lambda\left(\left[\Omega_{12}\right]-\left[\Omega_{12}\right]^{\dagger}\right) \omega
\end{gathered}
$$

\section{THE EXPERIMENTAL RESULTS AND ANALYSIS}

\subsection{Experimental area and the data}

In this paper we describe our experiments with high-resolution X-band full Polarimetric SAR data acquired by a dual-baseline interferometric airborne SAR system over an area of Danling in southern China. In this area the elevation difference is about 1000 meters, the average elevation is about 450 meters, and the experimental area include urban, rural, rivers, roads, vegetation and farmland, which are very suitable for DEM extraction under the different scattering mechanism research. The main image respectively at the four corner point coordinates are $30.01^{\circ}$, $103.53^{\circ}, 29.99^{\circ}, 103.57^{\circ}$. The line of image is 6041 and column is 16384 , the resolution is $0.3 \mathrm{~m}$, center angle of incidence is $44.17^{\circ}$, the baseline length is $2.19 \mathrm{~m}$. In order to verify various effects and test method for extraction of DEM precision, we make the field measurements of the control points as a reference.

\subsection{Data processing and analysis}

To illustrate the influence of polarization state for interferometric coherence, and also to facilitate the comparison results, first from full Polarimetric SAR data to select the single channel data for regular processing, then Pauli algorithm is used to generate the double polarimetric interferometry data, SVD, NR and PD methods are used to generate the full polarimetric interferometry data. Then we can make use of the polarimetric interferometric information to extract DEM, finally compared optimization results with the single polarimetric interferometry.

The DEM got by conventional polarization coherent interference, Pauli and optimization are shown in figure 2:

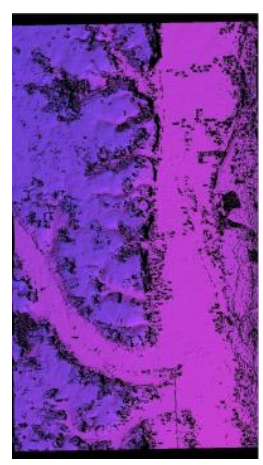

HH_HH

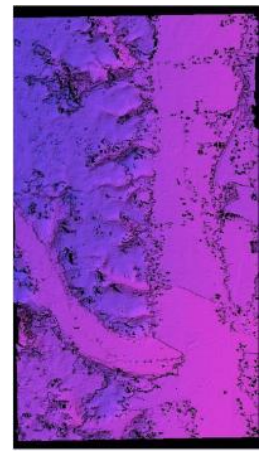

$\mathrm{HH}+\mathrm{VV}$

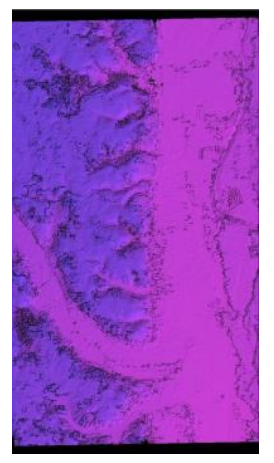

SVD1

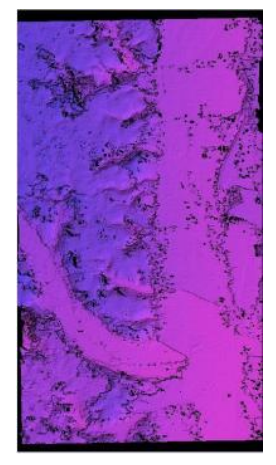

NR1

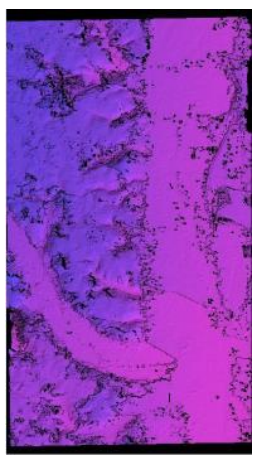

$\mathrm{HV}+\mathrm{VH}$

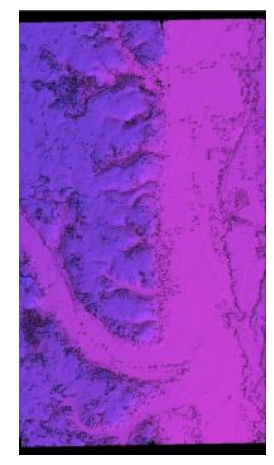

PD-Low

Figure 2. DEM

From the figure 2, the DEM of generated by Pauli processed is higher than the conventional single polarimetric interferometry, while the result of full polarimetric interferometry is the best. The table 2 shows that through the vector interference and coherent optimization can effectively suppress noise, and can reduce the number of residual, phase 
standard deviation is reduced greatly, it can improve the quality of the phase unwrapping and it can improve quality of DEM. Meanwhile, Even if the same number of polarimetric channel, different polarimetric channel and different optimization methods also can bring different results.

We choose typical HH polarization, Pauli of $\mathrm{HH}+\mathrm{HV}$ and SVD three methods to make DEM accuracy evaluation, we used the 13 GPS control points for the DEM elevation accuracy, table 1 is the DEM of three ways measured and GPS measured elevation, figure 3 and figure 4 is the line chart DEM elevation and DEM elevation difference.

\begin{tabular}{|c|c|c|c|c|}
\hline 点号 & $\begin{array}{c}\text { GPS 实测 } \\
\text { 高程 }\end{array}$ & HH & HH+HV & SVD \\
\hline $\mathrm{xd} 260030$ & 433.59300 & 429.961365 & 434.529633 & 434.637909 \\
\hline $\mathrm{xd} 260031$ & 431.38800 & 426.769989 & 430.005310 & 429.999054 \\
\hline $\mathrm{xd} 260032$ & 475.34500 & 471.306274 & 475.952209 & 476.171112 \\
\hline $\mathrm{xd} 260033$ & 480.08300 & 480.824219 & 480.952087 & 480.702271 \\
\hline $\mathrm{xd} 260034$ & 478.41600 & 478.062775 & 478.925751 & 478.660706 \\
\hline $\mathrm{xd} 260035$ & 427.20600 & 429.425293 & 426.877869 & 427.027802 \\
\hline $\mathrm{xd} 260036$ & 477.00200 & 476.398895 & 478.311768 & 478.217651 \\
\hline $\mathrm{xd} 260037$ & 427.46700 & 438.137085 & 427.063477 & 427.338776 \\
\hline $\mathrm{xd} 260038$ & 480.62700 & 487.379364 & 483.009125 & 480.789520 \\
\hline $\mathrm{xd} 260039$ & 420.59800 & 414.053101 & 420.906036 & 421.012848 \\
\hline $\mathrm{xd} 260040$ & 435.53400 & 430.136749 & 436.462585 & 436.682465 \\
\hline $\mathrm{xd} 260041$ & 470.80900 & 451.917755 & 473.009216 & 471.608124 \\
\hline $\mathrm{xd} 260042$ & 482.85100 & 487.022308 & 483.849091 & 482.514801 \\
\hline 均值 & 0 & -1.501833 & 0.687320 & 0.341849 \\
\hline 中误差 & 0 & 7.138624 & 1.197041 & 0.781614 \\
\hline
\end{tabular}

Table1 DEM elevation contrast

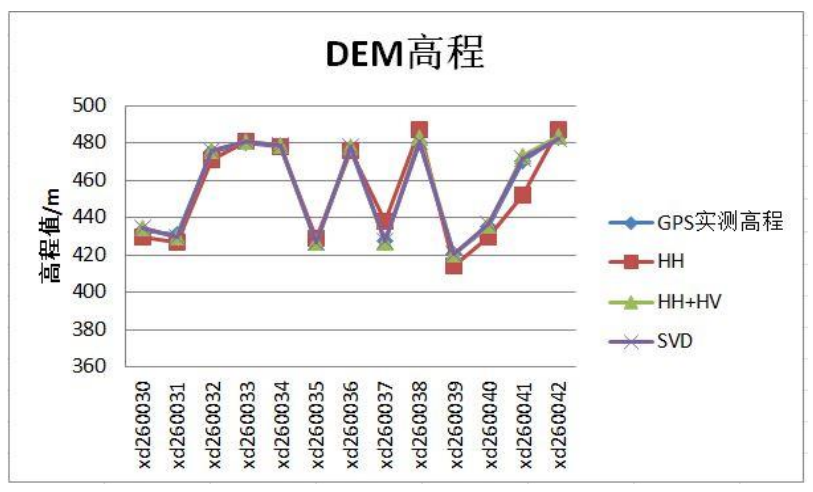

Figure 3. DEM elevation contrast

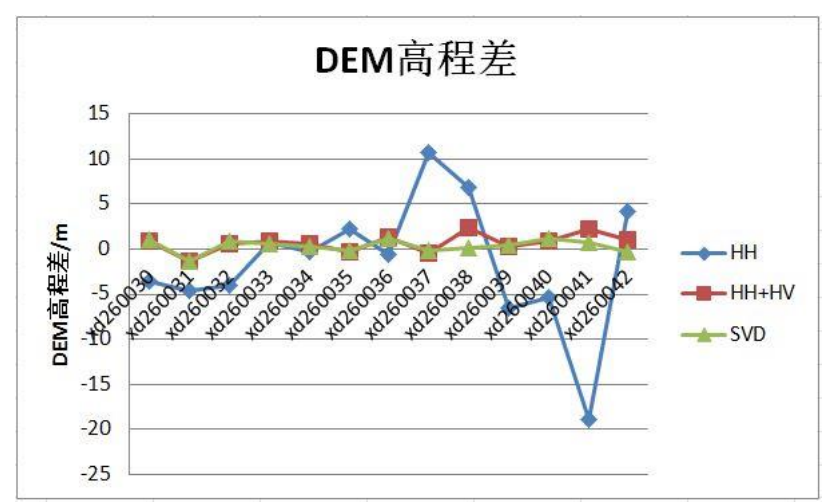

Figure 4. DEM elevation difference contrast

From the picture, it has been observed that optimization ways can greatly improve the precision of DEM, the method of Pauli can improve an average of about 3 to 4 meters compared to single polarimetric interferometry, and the optimal method of SVD can improve about 1 to 2 meters compared to the Pauli. But in specific places, such as 30 to 32 control points are located in broken terrain with a small amount of vegetation and the place of the building, the precision of DEM got by PolInSAR has a certain degree of increase than the single polarimetric interferometry, but the SVD has no significant change compared to the method of Pauli. The control points of 33to 36 locate in the relatively flat terrain, mostly low fields. Compared with the single polarimetric interferometry, polarization interferometry couldn't reflect the great advantage. The precision of DEM is basically the same. And in the area of 37 to 42 points with density trees or tall building, body scattering to coherent and geometry to coherent can restrict the reliability of generating the high precision DEM, especially body scattering to coherent caused by scattering center height difference in the resolution cell, while polarization coherent interference optimal process can effectively reduce the decorrelation caused by scattering center height difference in an effective resolution cell.SO in this area the precision of DEM got by PolInSAR has a great degree of increase than the single polarimetric interferometry, and the SVD also has a significant change compared to the method of Pauli. Particularly around 31 and 47 control points, a large number of vegetation and building make the coherence so poor and lead to phase unwrapping exist errors, at last in this area the single polarimetric interferometry can make the DEM elevation has about 10 meters error, while PolInSAR can obtain a correct measurements.

\section{CONCLUSION}

In this paper we describe our experiments with high-resolution X-band full Polarimetric SAR data acquired by a dual-baseline interferometric airborne SAR system over an area of Danling in southern China. Pauli algorithm is used to generate the double polarimetric interferometry data, Singular Value Decomposition (SVD), Numerical Radius (NR) and Phase diversity (PD) methods are used to generate the full polarimetric interferometry data. Finally compared optimization results with the single polarimetric interferometry, it has been observed that optimization ways can improve the precision of DEM. The result of full polarimetric interferometry is better than double polarimetric interferometry. Meanwhile, in different terrain, the result of full polarimetric interferometry will have a different degree of increase. The precision of DEM got by PolInSAR has no significant change than the single polarimetric interferometry 
in the relatively flat terrain, in the area with density trees or tall building the precision of DEM got by PolInSAR has a great degree of increase than the single polarimetric interferometry.

\section{ACKNOWLEDGEMENTS}

This work is supported by Special Fund for Surveying and Mapping and Geoinformation Research in the Public Interest (Grant No.201512022) and National Natural Science Foundation of China (Grant No.41401530).

\section{REFERENCES}

Cloude, S.R., Papathanassiou, K.P., Polarimetric optimization in radar interferometry. Electronics Letters, 33(13), p.11761178.1997.

Li X W, Guo H D, Wang C L, et al. Generation and Error Analysis of DEM Using Spaceborne Polarimetric SAR Interferometry Data[C]//IEEE International Geoscience and Remote Sensing Symposium (IGARSS'02), Toronto, Canada, 2002, 5:2705-707

Li Xinwu. Information Extraction Methods and Application Study of Polarimetric SAR Interferometry [D].Beijing: Institute of Remote Sensing Application, Chinese Academy of Sciences, 2002.

Xiong Tao, Yang Jian, Peng Yingning. Approach to Reversion of DEM based on Polarimetric SAR Interferometry [J].Journal Tsinghua University (Science \& Technology), 2007, 47(7):1170-1173.

Brandfass M.Generation of Bald Earth Digital Elevation Models as Applied to Polarimetri SAR Interferometry [A].IGARSS'02[C].2002.

Luciano V D, Marcos T E, el al. Assessment of Digital Elevation Models Obtained in Brazilian Amazon Based on $\mathrm{P}$ and $\mathrm{X}$ band Airborne Interferomatric Data[A]. IGARSS'02[C].2002.

Cloude, S.R., Papathanassiou, K.P., Three-stage inversion process for polarimrtric SAR interferometry. IEE Proceeding of Radar Sonar Navition, 150(3), p.125-134.2003.

Colin E, Titin-Schnaider C, Tabbara W. An Interferometric Coherence Optimization Method in Radar Polarimetry for High Resolution Imagery [J].IEEE Transactions on Geoscience and Remote Sensing,2006,44(1):167-175.

Tabb M, Orrey J, Flynn T, et al. Phase Diversity: A Decomposition for Vegetation Parameter Estimation Using Polarimetric SAR Interferimetry[C]/Proceedings of 4th European Synthetic Aperture Radar Conference,2002:721-724. 